\title{
Mitigation of transverse domain growth in two-dimensional polarization switching of lithium niobate
}

\author{
L.-H. Peng, ${ }^{\text {a) }}$ Y.-C. Shih, S.-M. Tsan, and C.-C. Hsu \\ Department of Electrical Engineering and Institute of Electro-Optical Engineering, National Taiwan \\ University, Taipei, Taiwan, Republic of China
}

(Received 20 June 2002; accepted 2 November 2002)

\begin{abstract}
This letter reports a charge control mechanism to mitigate the growth of transverse domain in the two-dimensional periodical polarization switching of $Z$-cut congruent-grown lithium niobate. This mechanism is established in a two-step procedure consisting of a first high temperature treatment followed by pulsed field poling. The ferroelectric $\chi^{(2)}$ nonlinear photonic crystal thus formed exhibits a domain size as small as $3.3 \mu \mathrm{m}$ and can emit an array of second harmonic green lasers when pumped by a Nd:yttritium-aluminum-garnet laser. These observations are ascribed to the (i) selective $180^{\circ}$ domain nucleation underneath the oxidized electrode, and (ii) confinement of transverse domain motion to the positively charged boundary formed by the heat treatment. (C) 2002 American Institute of Physics. [DOI: 10.1063/1.1533115]
\end{abstract}

The ferroelectric crystals are known for their abundant acoustic, mechanical, and electro-optical properties that make them an attractive area of further study in condensed matter physics. ${ }^{1}$ The feature for ferroelectric domain to reverse its polarization $\left(P_{s}\right)$, i.e., the so-called polarization switching or domain reversal, and the capability to retain a structure with alternative polarization states can substantially modify the material's tensor properties, thus resulting in device applications. One such example takes advantage of the reversible polarization and fast switching time to realize high-density memory devices for data storage. ${ }^{2}$ Another promising application for energy storage and charge amplification can be found on polarization gradient devices. ${ }^{3}$ When a ferroelectric superlattice is made of periodically reversed $P_{s}$ at every coherent length $l_{c}=\lambda_{\omega} / 4\left(n_{2 \omega}-n_{\omega}\right)$, one can encounter a superior conversion efficiency in the nonlinear wave generation. ${ }^{4}$ Moreover, as the reversed domain resides in a two-dimensional (2D) lattice structure, ${ }^{5}$ i.e., in the so-called $\chi^{(2)}$ nonlinear photonic crystal, one can further sustain phase matching processes that are absent in bulk crystals. With these accounts, it is pertinent to establish a polarization switching mechanism that can allow the manipulation of polarization switching at a desirable dimensionality and configuration.

To initiate a polarization switching, conventional wisdom suggests one can apply an electric field across the polar axis to overcome the coercive field $\left(E_{c}\right)$ in ferroelectric. ${ }^{6} \mathrm{An}$ external supply of the switching current, i.e., $2 A d P_{s} / d t$, serves to compensate the depolarization field in the newly switched area $A$. The distribution of switching current and charged field therefore plays an important role in determining the spatial arrangement of the reversed domains. ${ }^{7}$ Referring to Fig. 1, a commonly adapted procedure is to use a thick insulating layer such as photoresist (PR) to impede the mishap of current conduction in the unpatterned regime. ${ }^{8}$ However, electrostatic action from the charged domain wall

${ }^{a)}$ Electronic mail: peng@cc.ee.ntu.edu.tw and nucleation step constitutes a concern in the issue of domain broadening. ${ }^{9}$

To demonstrate an example of the electrostatic effects, we illustrate in Fig. 1 the distribution of horizontal switching field $E_{y}$ at a finite depth $(z=0.05 \Lambda$ where $\Lambda$ is the grating period) below the $+C$ surface of lithium niobate $\left(\mathrm{LiNbO}_{3}\right)$. Note this type of poling scheme has been recently attempted to fabricate $\chi^{(2)}$ nonlinear photonic crystal on $\mathrm{LiNbO}_{3} \cdot{ }^{10-12}$ Our evaluation of $E_{z}$ and $E_{x(y)}$ are based on a finite difference method capable of solving the Poisson equation with space charge distribution and discontinuity on $P_{s} .{ }^{13}$ We first draw attention to a relatively large $E_{y} \sim 4 E_{c}$ encountered at the sharp interface between the poling electrode and the insulating layer. This observation can be traced back to the fringing field effect due to a large dielectric discontinuity $\left(\varepsilon_{r}=30\right.$ in $\mathrm{LiNbO}_{3}$ vs 3 in PR) in the electrostatic response. ${ }^{7,14}$ The appearance of switching field distortion indeed bears critical consequence on the kinetics of polarization switching. According to the Miller-Weinreich theory,

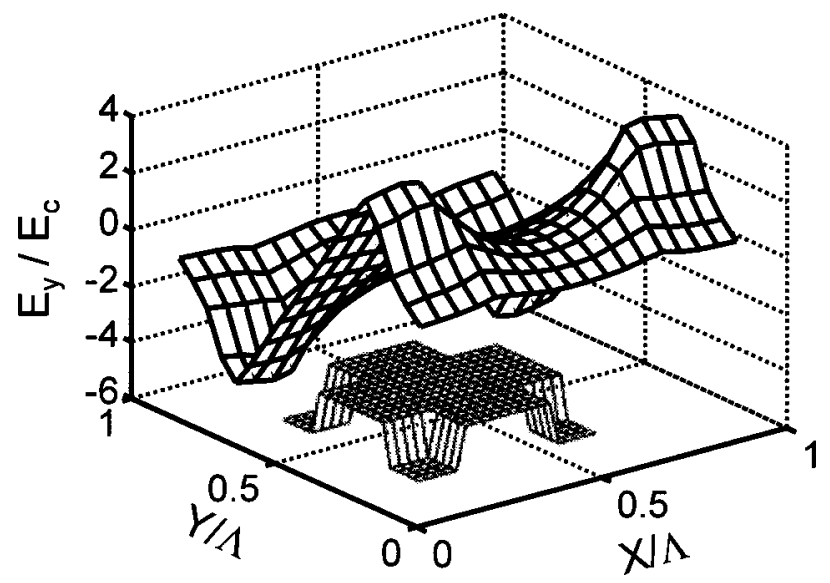

FIG. 1. Calculated distribution of $E_{y}$ at a depth $z=0.05 \Lambda$ below the $+C$ face of $\mathrm{LiNbO}_{3}$. The poling voltage is $10.5 \mathrm{kV}$ across a $500-\mu$ m-thick substrate. Inset: conventional poling configuration showing the use of thick $\mathrm{PR}$ layer to mask the unpoled region. The device periodicity is 6.6 $\times 6.6 \mu \mathrm{m}^{2}$ and the area of poling is $3.3 \times 3.3 \mu \mathrm{m}^{2}$. 


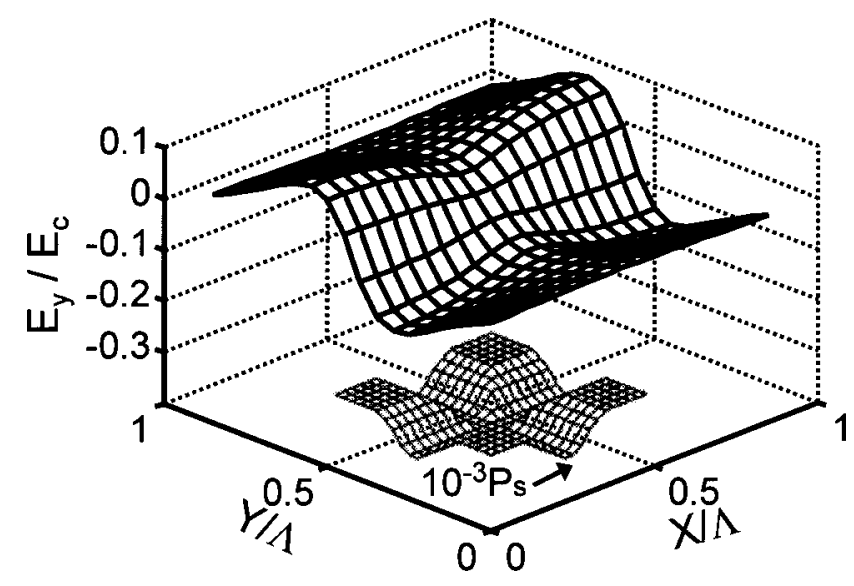

FIG. 2. Calculated distribution of $E_{y}$ at a depth $z=0.05 \Lambda$ along the positively charged boundary. Inset: a positively charged parabola of $10^{-3} P_{s}$ is placed at a maximum depth of $0.25 \Lambda$ between each pair of the poling electrodes.

the ferroelectric polarization switching exhibits a nucleation rate dependence on $\exp (-$ const $/ E)$. ${ }^{15}$ The drastic increase of $E_{z}$ above $E_{c}$ at the electrode edge (not shown) ensures the nucleation of $180^{\circ}$ domains and their fast forward growth down to the opposite side of the substrate. ${ }^{16}$ However, the sidewise expansion of inverted domain will be largely affected by the disturbance of tangential field. A close examination of Fig. 1 indicates the field line of $E_{y}$ (and $E_{x}$ ) acts to spread the switching current off the poling electrode. This effect introduces fast carrier redistribution underneath the insulating layer and has been ascribed to the source of causing domain irregularity and broadening in the unpatterned region. ${ }^{17}$

Albeit the tangential field only resides in a thickness $z$ $\sim \Lambda$ and fades away at larger distance, a good strategy to preserve the domain fidelity would be to suppress the inhomogeneous electrostatic response. This has been done by using low duty-cycle electrodes and thin substrates. ${ }^{18}$ Domain engineering, however, can still be impeded by crystal imperfections such as point defects ${ }^{19}$ and trapped charges. ${ }^{20} \mathrm{Cau}-$ tion must be exercised to avoid the backswitching of inverted domain when one terminates the field poling process on potassium titanyl phosphate (KTP), ${ }^{21} \mathrm{LiNbO}_{3},{ }^{22}$ and memory devices. ${ }^{23}$

In this work, we report a charge control mechanism to mitigate the transverse domain growth in the $2 \mathrm{D}$ polarization switching process on $\mathrm{LiNbO}_{3}$. The idea is to seek an electrostatic compensation over the tangential field prior to initiate electrical poling. In order to convey the polarization switching onto a designated lattice site, we consider the use of potential barrier to restrain the lateral $180^{\circ}$ domain motion. One such example is illustrated in Fig. 2 upon which a positively charged parabola of $10^{-3} P_{s}$ is placed between each pair of the poling electrodes. We note the replacement of a thick insulating layer by a positively charged barrier can substantially reduce the tangential field by one order of magnitude down to $0.1 E_{c}$ in the peak value. In addition, we now have the field line of $E_{x}\left(E_{y}\right)$ in Fig. 2 pointing to an outward direction underneath the unpatterned region. This observation signifies a promising mechanism to oppose the current spreading effect and would be valuable for the control of transverse domain motion.

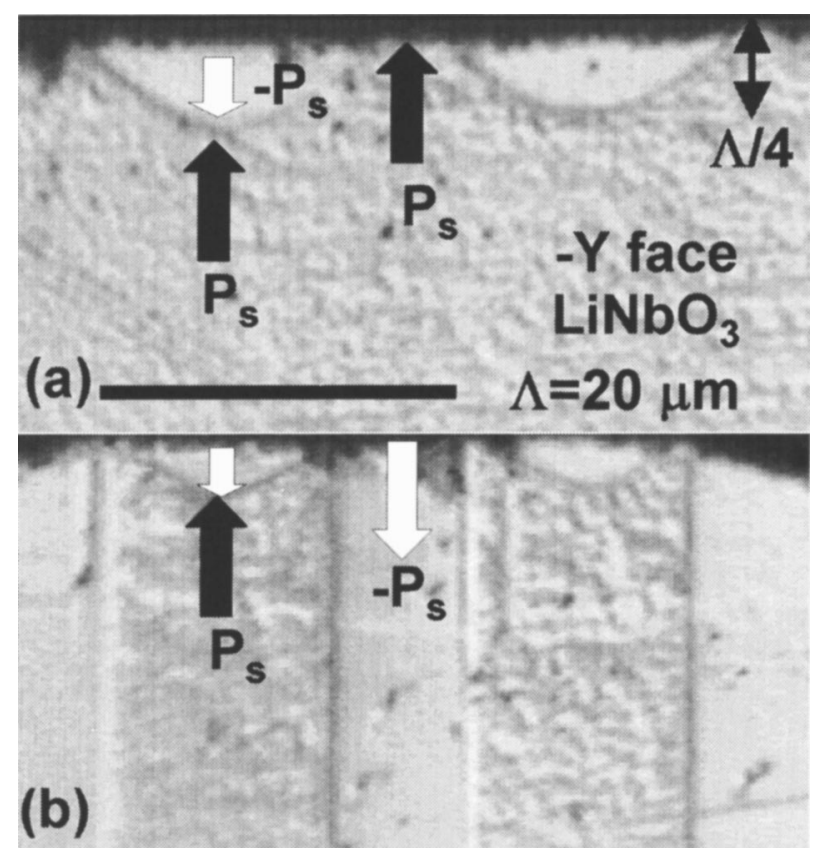

FIG. 3. $-Y$ face micrographs of $\mathrm{LiNbO}_{3}$ with a $20 \mu \mathrm{m}$ grating period in (a) after the first high-temperature treatment at $1050^{\circ} \mathrm{C}$, and (b) continued with poling at $21 \mathrm{kV} / \mathrm{mm}$. The substrate thickness is $500 \mu \mathrm{m}$.

Based upon the earlier scenario, polarization switching was performed on 500- $\mu$ m-thick, $Z$-cut congruent-grown $\mathrm{LiNbO}_{3}$ substrates purchased from Crystal Technology, USA. A thin $(\sim 50 \mathrm{~nm})$ aluminum (Al) electrode pattern was evaporated onto the $+C$ face of $\mathrm{LiNbO}_{3}$ using a standard lithography technique. To engage a positive charge distribution, we take advantage of the divergence effect on $P_{s}$, i.e., $-\nabla \cdot P_{s}=\rho$. This can be achieved in a high temperature process ${ }^{24}$ by placing the patterned sample inside a quartz tube furnace and undergoing a heat treatment at $1050^{\circ} \mathrm{C}$ for $5 \mathrm{~h}$ in ambient air. The sample was then transferred to a poling apparatus consisting of a pulsed voltage amplifier made from Trek (model 20/20A for $20 \mathrm{kV}$ and $20 \mathrm{~mA}$ output) and using lithium chloride liquid electrode to establish an electric contact to $\mathrm{LiNbO}_{3} .{ }^{25}$ Details of the poling procedure can be found in Ref. 26

In supporting our model analysis of charge control mechanism, a one-dimensional periodically poled example has been demonstrated. Illustrated in Fig. 3 are the etched $-y$ face micrographs of $\mathrm{LiNbO}_{3}$ with a $20 \mu \mathrm{m}$ period in (a) after the first high-temperature treatment, and (b) continued with an electrical poling at $21 \mathrm{kV} / \mathrm{mm}$. In the unpatterned region open to the air, we encounter the presence of triangularly inverted domains which can extend as deep as $0.25 \Lambda$ $(5 \mu \mathrm{m})$. This can bee seen from the etch rate difference with respect to the virgin crystal. This phenomenon is in contrast to a recent study on $\mathrm{SiO}_{2}$ coated $\mathrm{LiNbO}_{3}{ }^{27}$ and $\mathrm{KTP}^{28}$ upon which the piezoelectric effect is credited to the cause of domain inversion. Such observations suggest the origin of surface domain inversion in the present study is dominated by the thermal induced outdiffusion process. ${ }^{29-31}$ Note a drastic change in the surface composition could introduce a severe variation in the coercive field. This mechanism could therefore result in a transverse domain growth sensitive to the composition inhomogeneity.

On the other hand, we have found the micro-porosity 


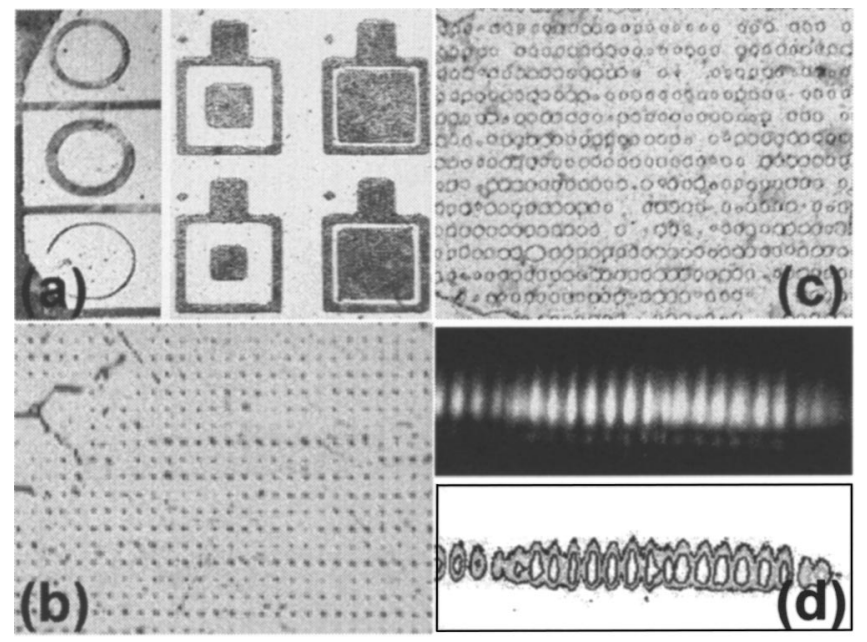

FIG. 4. $-Z$ face micrographs of $\mathrm{LiNbO}_{3}$ showing inverted domains of (a) ring- and hollow-square shape, (b) grid-, and (c) cylinder-like periodically poled structures with periodicity of $15 \times 20$ and $6.6 \times 13.2 \mu \mathrm{m}^{2}$, and (d) CCD image and intensity of far field pattern from the emission of arrayed QPM-SHG green laser of (c).

formed in the oxidized $\mathrm{Al}_{2} \mathrm{O}_{3}$ electrode of heat treatment can selectively establish an electric contact to the underlying $\mathrm{LiNbO}_{3}$ and provide a current limiting mechanism to the polarization switching process. ${ }^{32}$ Further evidence is provided in Fig. 3(b) from which the nucleation of $180^{\circ}$ domains is found to take place only underneath the oxidized $\mathrm{Al}_{2} \mathrm{O}_{3}$ electrode. The most inspiring phenomenon, however, is that the electrically poled $180^{\circ}$ domain and its transverse motion are confined to the edge of triangular domain boundary. Such observations clarify the use of positively charged barriers to inhibit the spreading of switching current and to constrain the growth of transverse domain in the periodical poling process.

Further shown in Fig. 4(a) are the imprinting and retaining of $2 \mathrm{D}$ inverted domains of ring- and hollow squarepattern on the $-Z$ face of $\mathrm{LiNbO}_{3}$. The domain wall thickness can be as small as $10 \mu \mathrm{m}$. We also illustrate in Fig. 4 the periodically poled 2D lattice structure with (b) grid- and (c) cylinder-like domain pattern whose periodicity is $15 \times 20$ and $6.6 \times 13.2 \mu \mathrm{m}^{2}$, respectively. The lightly etched regions, now appearing as a surface-relief on the $-Z$ face of $\mathrm{LiNbO}_{3}$, reveal the versatility of domain shape (rectangular or hexagonal) and size (as small as $3.3 \mu \mathrm{m}$ ) control that can be resultant from the charge control mechanism. We further note that the $500-\mu \mathrm{m}$-thick, cylinder-like $2 \mathrm{D}$ periodically poled $\mathrm{LiNbO}_{3}$ rectangular lattice of Fig. 4(c) can indeed be quasiphase-matched (QPM) to the second-harmonic generation (SHG) when pumped by a $1064 \mathrm{~nm} \mathrm{Nd:yttritium-}$ aluminum-garnet laser (Laser Quantum, UK). The charge coupled device (CCD) image and intensity distribution shown in Fig. 4(d) are the first demonstration of arrayed 2D-QPM SHG of continuous wave green lasers of its kind. Detailed device characterization of this $2 \mathrm{D} \chi^{(2)}$ nonlinear photonic crystal will be presented in a forthcoming publication.

In summary, we report an electrostatic control mechanism to mitigate the transverse domain motion in the polarization switching process of $Z$-cut congruent-grown $\mathrm{LiNbO}_{3}$. It is shown the use of positively charged potential barriers, which arises from $-\nabla \cdot P_{s}=\rho$ in a preset heat treatment, can substantially inhibit the spreading of switching current and restrain the $180^{\circ}$ domain motion. These observations are ascribed to the constraint of $180^{\circ}$ domain nucleation in the oxidized electrode and confinement of transverse domain growth by the charged boundary.

The authors wish to acknowledge technical assistance from Professor W.-S. Wang and the Integrated Optics and Solid State Laboratories at NTUEE. This research was supported by the National Science Council, Grant No. 90-2215E-002-017 and 023.

${ }^{1}$ For a recent review, please see Ferroelectric and dielectric thin films in Handbook of Thin Film Materials, Vol. 3 and Handbook of Advanced Electronic and Photonic Materials and Devices, Vol. 4, edited by H. S. Nalwa (Academic, New York, 2002 and 2001).

${ }^{2}$ O. Auciello, J. F. Scott, and R. Ramesh, Phys. Today 51, 22 (1998).

${ }^{3}$ J. V. Mantese, N. W. Schubring, and A. L. Micheli, Appl. Phys. Lett. 80, 1430 (2002)

${ }^{4}$ J. A. Armstrong, N. Bloembergen, J. Ducuing, and P. S. Pershan, Phys. Rev. 127, 1918 (1963).

${ }^{5}$ V. Berger, Phys. Rev. Lett. 81, 4136 (1998).

${ }^{6}$ J. Camlibel, J. Appl. Phys. 40, 1690 (1969).

${ }^{7}$ G. Rosenman, Kh. Garb, A. Skliar, M. Oron, D. Eger, and M. Katz, Appl. Phys. Lett. 73, 865 (1998).

${ }^{8}$ G. D. Miller, R. G. Batchko, W. M. Tulloch, D. R. Weise, M. M. Fejer, and R. L. Byer, Opt. Lett. 22, 1834 (1997).

${ }^{9}$ V. Ya. Shur, E. L. Rumyantsev, E. V. Nikolaeva, and E. I. Shishkin, Appl. Phys. Lett. 77, 3636 (2000).

${ }^{10}$ N. G. R. Broderick, G. W. Ross, H. L. Offerhaus, D. J. Richardson, and D. C. Hanna, Phys. Rev. Lett. 84, 4345 (2000).

${ }^{11}$ A. Chowdhury, C. Status, B. F. Boland, T. F. Kuech, and L. McCaughan, Opt. Lett. 26, 1353 (2001).

${ }^{12}$ S. M. Russell, P. E. Powers, M. J. Missey, and K. L. Schepler, IEEE J. Quantum Electron. 37, 877 (2001).

${ }^{13}$ L. Baudry and J. Tournier, J. Appl. Phys. 90, 1442 (2001).

${ }^{14}$ J. D. Jackson, Classical Electrodynamics, 2nd ed. (Wiley, New York, 1975).

${ }^{15}$ R. C. Miller and G. Weinreich, Phys. Rev. 117, 1460 (1960).

${ }^{16}$ G. D. Miller, Ph.D. thesis, Stanford University, 1998.

${ }^{17}$ P. Urenski, M. Molotskii, and G. Rosenman, Appl. Phys. Lett. 79, 2964 (2001).

${ }^{18}$ K. Mizuuchi and K. Yamamoto, Appl. Phys. Lett. 66, 2943 (1995).

${ }^{19}$ W. Jo, D. C. Kim, and J. W. Hong, Appl. Phys. Lett. 76, 390 (2000).

${ }^{20}$ A. Roelofs, N. A. Pertsev, R. Waser, F. Schlaphof, L. M. Eng, C. Ganpule, V. Nagarajan, and R. Ramesh, Appl. Phys. Lett. 80, 1424 (2002).

${ }^{21}$ J. Hellström, R. Clemens, V. Pasiskevicius, H. Karlsson, and F. Laurell, J. Appl. Phys. 90, 1489 (2001).

${ }^{22}$ R. G. Batchko, V. Y. Shur, M. M. Fejer, and R. L. Byer, Appl. Phys. Lett. 75, 1673 (1999).

${ }^{23}$ J. F. Scott, Ferroelectric Memories (Springer, New York, 2001).

${ }^{24}$ K. Nakamura, H. Ando, and H. Shimizu, Appl. Phys. Lett. 50, 1413 (1987).

${ }^{25}$ L. E. Myers, R. C. Eckardt, M. M. Fejer, R. L. Byer, W. R. Bosenberg, and J. W. Pierce, J. Opt. Soc. Am. B 12, 2102 (1995).

${ }^{26}$ L.-H. Peng, Y.-C. Zhang, and Y.-C. Lin, Appl. Phys. Lett. 78, 4 (2001).

${ }^{27}$ M. Fujimura, T. Suhara, and H. Nishihara, Electron. Lett. 27, 1207 (1991).

${ }^{28}$ K. S. Buritskii, E. M. Dianov, V. A. Maslov, V. A. Chernykh, and E. A. Shcherbakov, J. Appl. Phys. 79, 3345 (1996).

${ }^{29}$ J. Webjörn, F. Laurell, and G. Arvidsson, IEEE Photonics Technol. Lett. 1, 316 (1989).

${ }^{30}$ V. D. Kugel and G. Rosenman, Appl. Phys. Lett. 62, 2902 (1993).

${ }^{31}$ L. Huang and N. A. F. Jaeger, Appl. Phys. Lett. 65, 1763 (1994).

${ }^{32}$ L.-H. Peng, Y.-J. Shih, and Y.-C. Zhang, Appl. Phys. Lett. 81, 1666 (2002). 
Applied Physics Letters is copyrighted by the American Institute of Physics (AIP). Redistribution of journal material is subject to the AIP online journal license and/or AIP copyright. For more information, see http:/ojps.aip.org/aplo/aplcr.jsp

Copyright of Applied Physics Letters is the property of American Institute of Physics and its content may not be copied or emailed to multiple sites or posted to a listserv without the copyright holder's express written permission. However, users may print, download, or email articles for individual use. 